Case report

https://www.journal-imab-bg.org

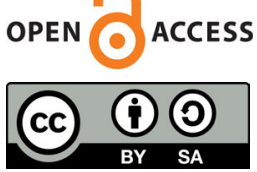

\title{
MODIFIED SINGLE ROLL FLAP APPROACH FOR SIMULTANEOUS IMPLANT PLACEMENT AND GINGIVAL AUGMENTATION
}

\author{
Kamen Kotsilkov \\ Department of Periodontology, Faculty of Dental Medicine, Medical University \\ - Sofia, Bulgaria.
}

\begin{abstract}
:
INTRODUCTION: The frequent result of tooth extraction is the resorption of the buccal alveolar wall and formation of I class (Siebert) alveolar ridge defect.

Another frequent problem in the implant surgery after the elevation of the standard full thickness flap is the choice of the exact vertical implant position to provide adequate biological width with $3 \mathrm{~mm}$ keratinized soft tissue above a bone level implant.

Nowadays the aim is to develop minimally invasive surgical approaches for other surgical applications.

AIM: This presentation demonstrates the application of a newly modified, minimally invasive, single roll flap approach for simultaneous implant placement and gingival augmentation.

MATERIALS AND METHODS: A 47 years old patient presented with fractured left central incisor. A new surgical technique for simultaneous implant placement and gingival augmentation was applied. Buccal partial thickness flap was prepared with de-epithelization and roll flap elevation of the crestal keratinized tissue, thus forming new papillae and ensuring implant placement in the exact vertical position. After one week of healing the final screw retained restoration was placed.

RESULT: The application of this newly developed by the author modification of the surgical protocol allowed precise implant placement consistent with the biological width requirements and simultaneously achieving buccal gingival augmentation.

CONCLUSION: The proposed new surgical approach provides an opportunity for minimally invasive implant placement without periosteal elevation. The modified roll flap preserves the keratinized tissues of the alveolar ridge using them to provide adequate buccal soft tissue volume. Furthermore, this approach ensures precise selection of the optimal implant depth, thus providing maximal functional and aesthetic result.
\end{abstract}

Keywords: single flap implant placement, modified roll flap, soft tissue oriented implant positioning.

\section{INTRODUCTION:}

A frequent result of tooth extraction is the resorption of the buccal alveolar wall and formation of I class (Siebert) alveolar ridge defect. Therefore a gingival augmentation with connective tissue graft is often required along with the implant placement for the correction of the bone defect, especially in regions with high aesthetic importance. However, this approach requires a second palatal surgical area which increases the surgical trauma and leads to higher risk of patient discomfort.

Another frequent problem in the implant surgery after the elevation of the standard full thickness flap is the choice of the exact vertical implant position to provide adequate biological width with $3 \mathrm{~mm}$ keratinized soft tissue above a bone level implant.

The contemporary approaches in periodontology rely on minimally invasive surgical protocols, aimed at complete tissue preservation in order to achieve and maintain primary closure and at stimulating the natural regenerative potential of the tissues. Nowadays the aim is to develop similar approaches for other surgical applications.

\section{MATERIALS AND METHODS:}

This presentation demonstrates the application of a new, minimally invasive surgical approach for simultaneous implant placement and gingival augmentation.

A 47 years old patient presented with a fractured left central incisor (Fig.1.)

Fig. 1. Initial clinical status.

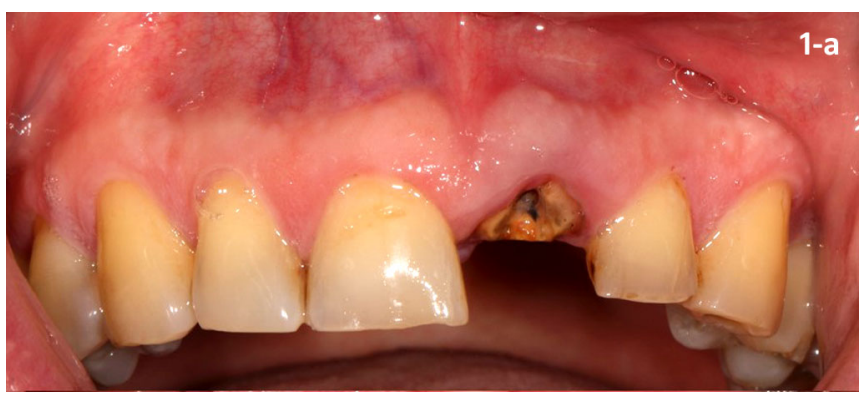




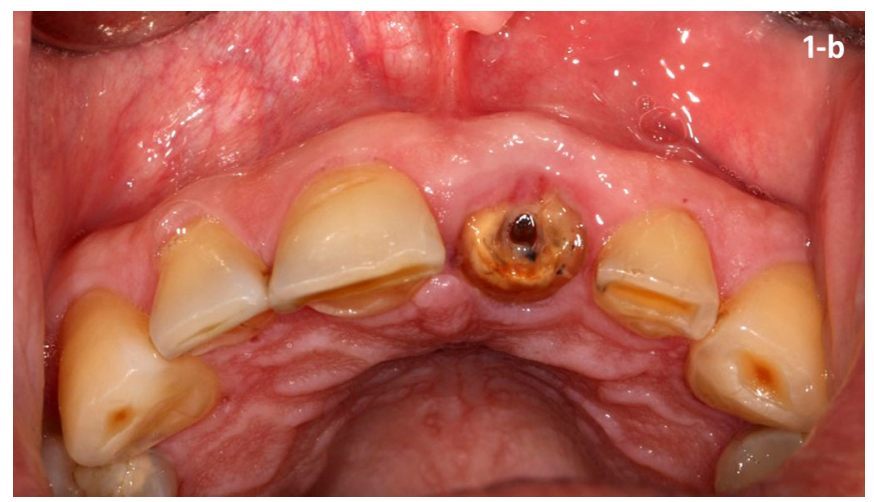

The clinical examination showed along with the missing clinical crown of the incisor, a vertical root fracture. The probing depth buccally at the level of the fracture was $14 \mathrm{~mm}$.

The radiographic analysis with a Cone Beam Computed Tomography revealed a deep buccal bone dehiscence with an almost complete destruction of the buccal wall of the tooth socket. The planning of the most appropriate implant position showed a limited possibility of initial implant anchorage to the bone (Fig. 2.).

Fig. 2. CBCT analysis

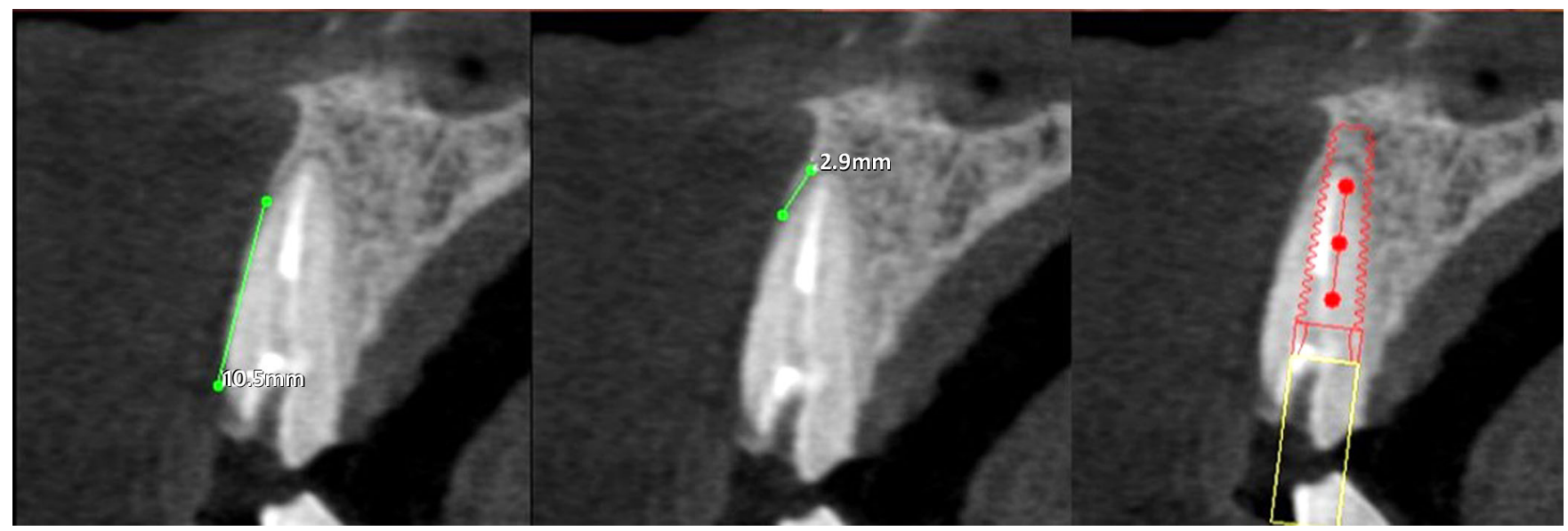

The analysis of the clinical situation determined an unpredictable outcome for an immediate implant placement due to the risk of low initial implant stability and the high probability of post-extractional soft tissue recession because of the lacking of the buccal bone plate. Therefore a staged approach with atraumatic tooòh extraction and early implant placement was chosen.

The first procedure included an atraumatic root extraction, keeping the integrity of the interdental papillae (Fig. 3.)

Fig. 3. Atraumatic tooth extraction.

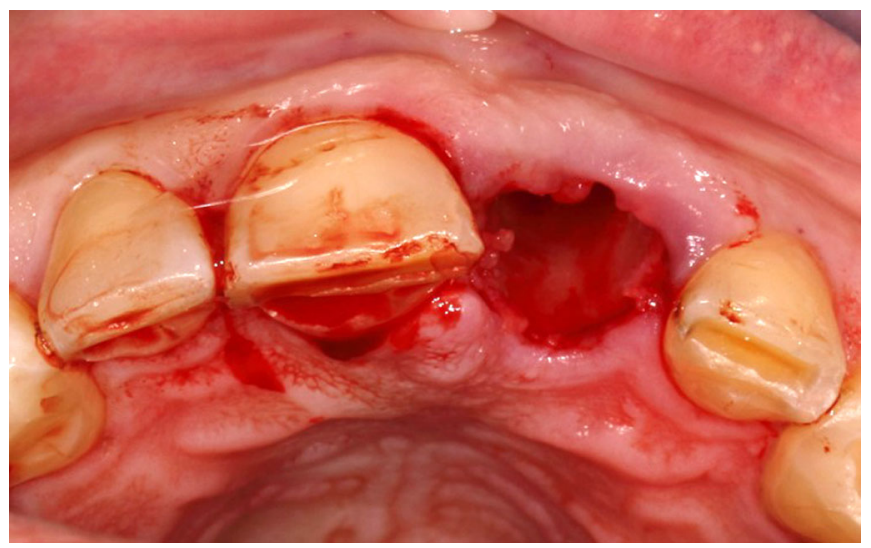

The blood clot in the tooth socket was protected with a collagen sponge, trimmed to size and fixated with cyanoacrylate tissue glue.

After 6 weeks healing period a complete soft tissue maturation was observed (Fig. 4.). During the healing process a mild $(<3 \mathrm{~mm})$ Siebert Class I defect of the alveolar ridge was formed due to the resorption of the buccal alveolar wall.

Fig. 4. Soft tissue healing 6 weeks post extraction.

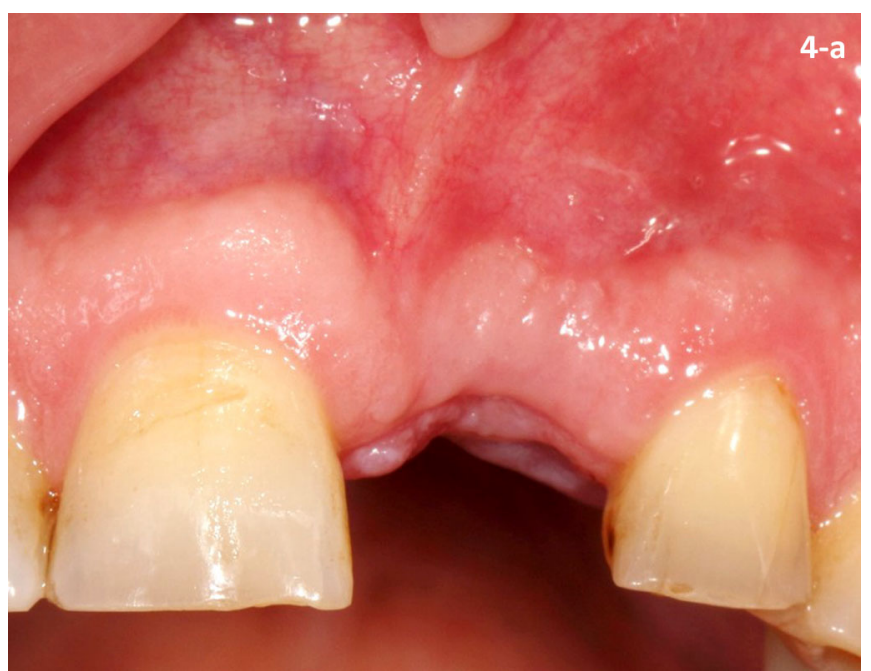




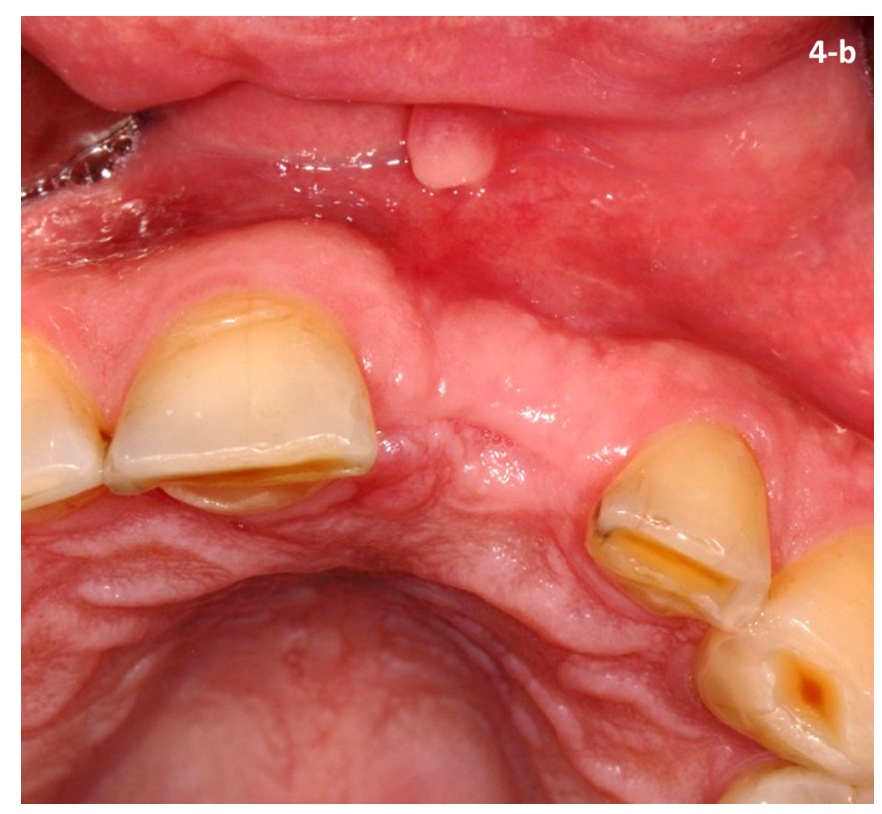

4-b
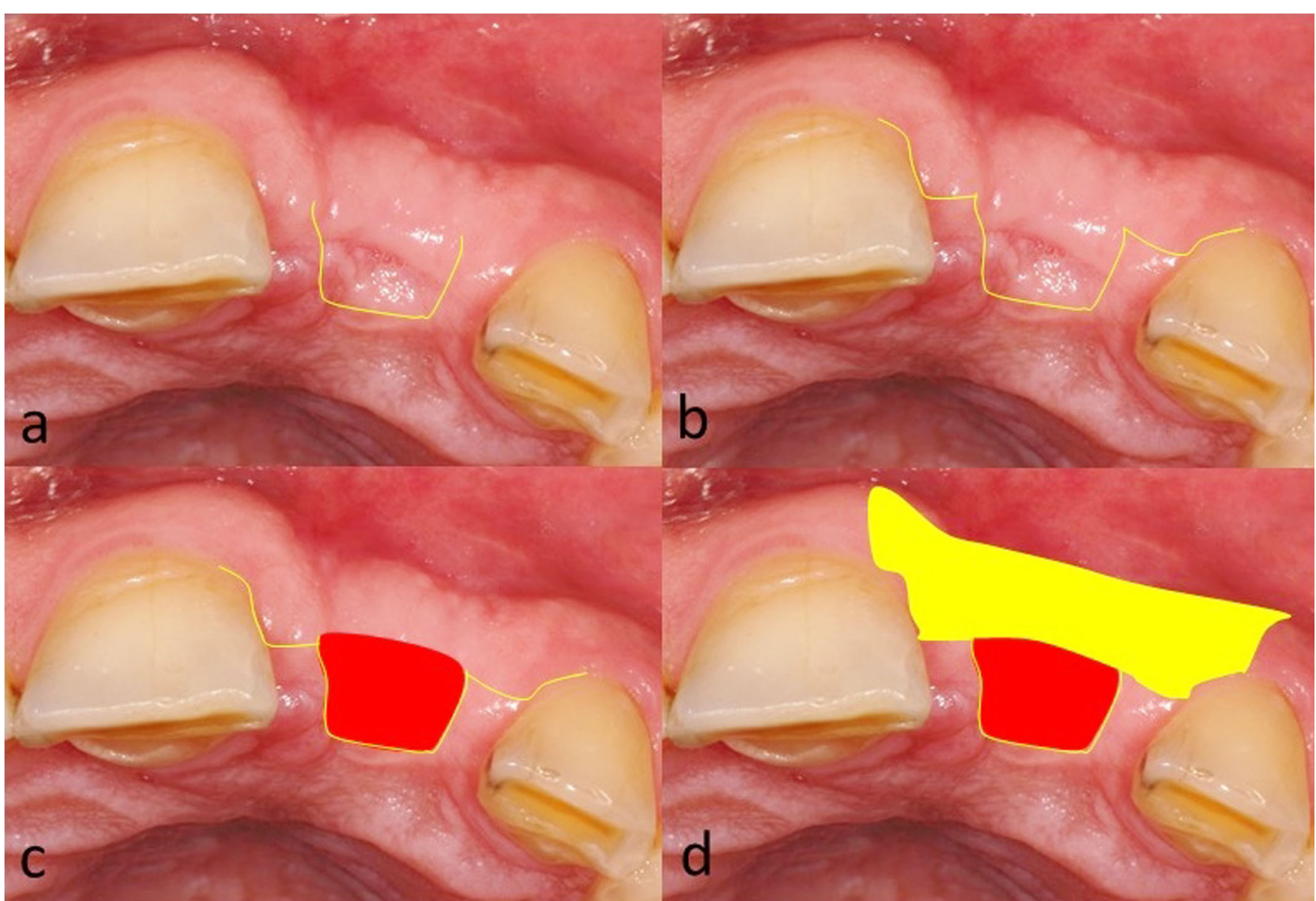

The finished flap preparation is presented on Fig. 6 . This new, minimally invasive design with split thickness preparation and lack of vertical releasing incisions provided sufficient flap vascularization and avoidance of scar formation on the buccal surface. 
Fig. 6. Finished flap preparation.

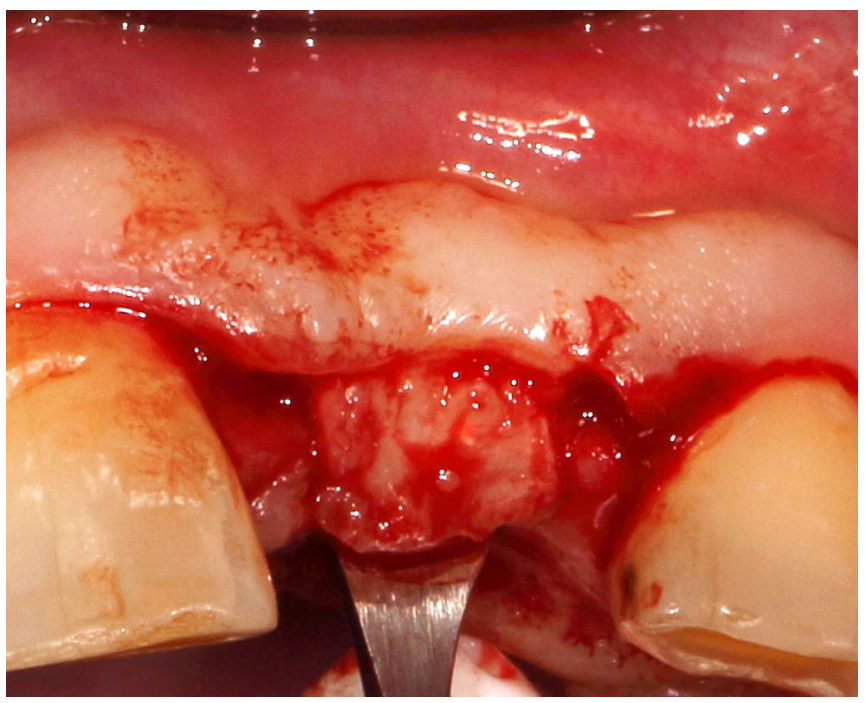

The flap design also ensured accurate three-dimensional implant position compliant with the desired emergency profile of the future prosthetic restoration. The mesiodistal and orofacial positioning was set with the separation of the U-shaped initial incision, and the coronoapical implant level was defined from the height of the recreated interdental papillae in order to ensure adequate biological width and proper soft tissue volume for the restoration (Fig. 7).
Fig. 7. Soft tissue oriented implant placement (Straumann BLT 4,1/12mm).

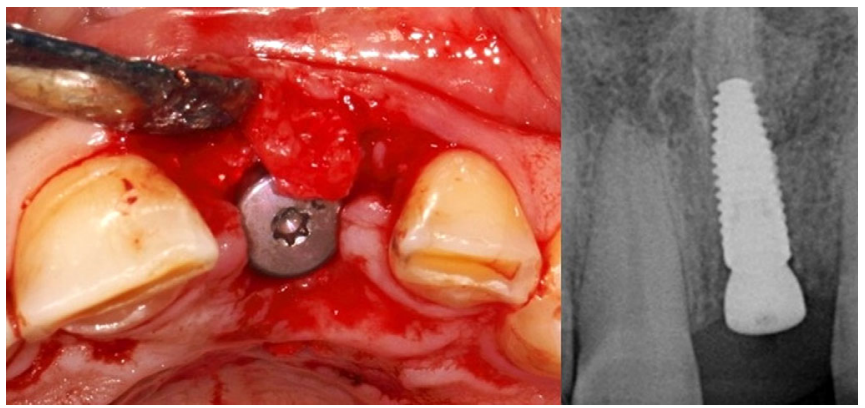

The deepithelized crestal flap was folded buccaly and inserted between the inner surface of the buccal flap and the preserved periosteum layer on the buccal wall of the residual socket, then it was sutured with a horizontal mattress suture to the buccal flap. The bucall flap was then advanced in a coronal position and sutured to the deepithelized recreated anatomical papillae. The avoided elevation of the papillae and the palatal tissues granted stable flap anchorage, thus ensuring primary intention healing. (Fig. 8).

Fig. 8. Flap advancement and suturing.

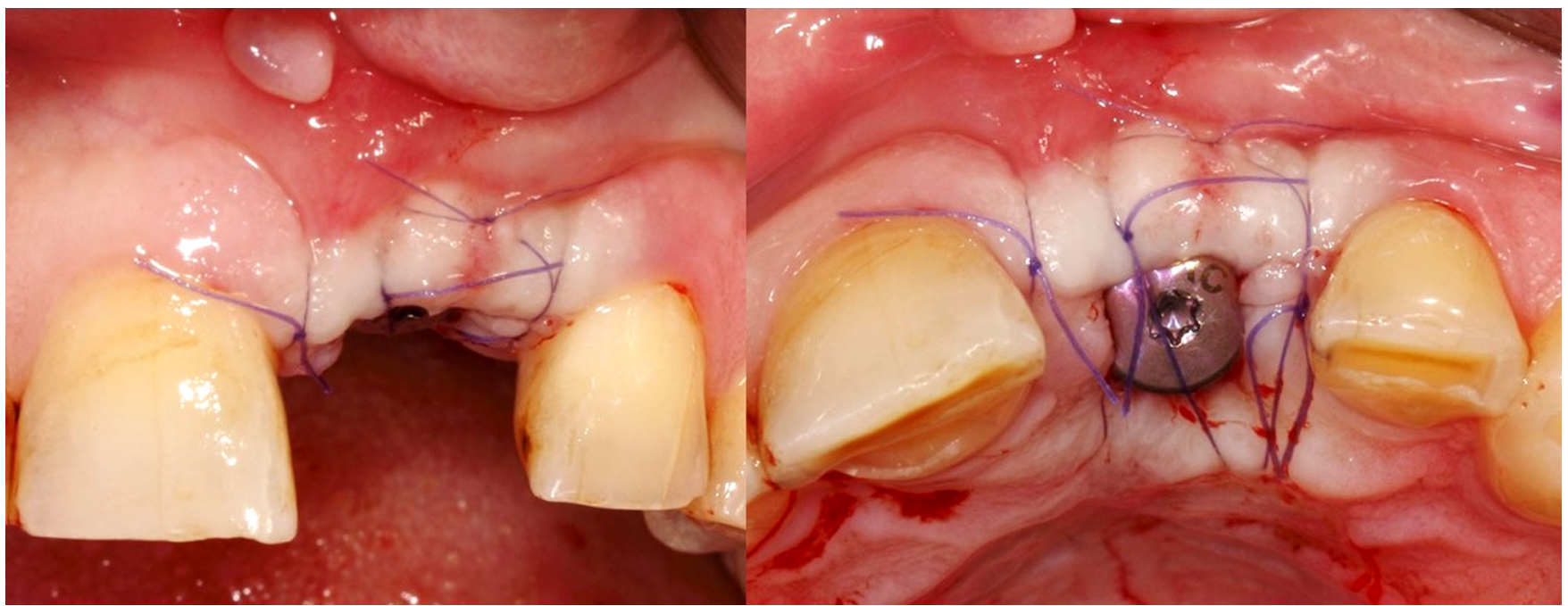

After an uneventful initial healing process, the sutures were removed on the 10th day. The tissue appearance suggested an advanced maturation process, which could be due to the minimally invasive flap preparation. The applied new flap design led to proper scalloping of the gingival margin corresponding to those on the adjacent teeth (Fig. 9a.) The occlusal view of the edentulous area demonstrated successful horizontal ridge augmentation (Fig. 9b) with enough soft tissue coronally to the implant level to provide proper restoration.(Fig. 9c). 
Fig. 9. Result at 10 days.

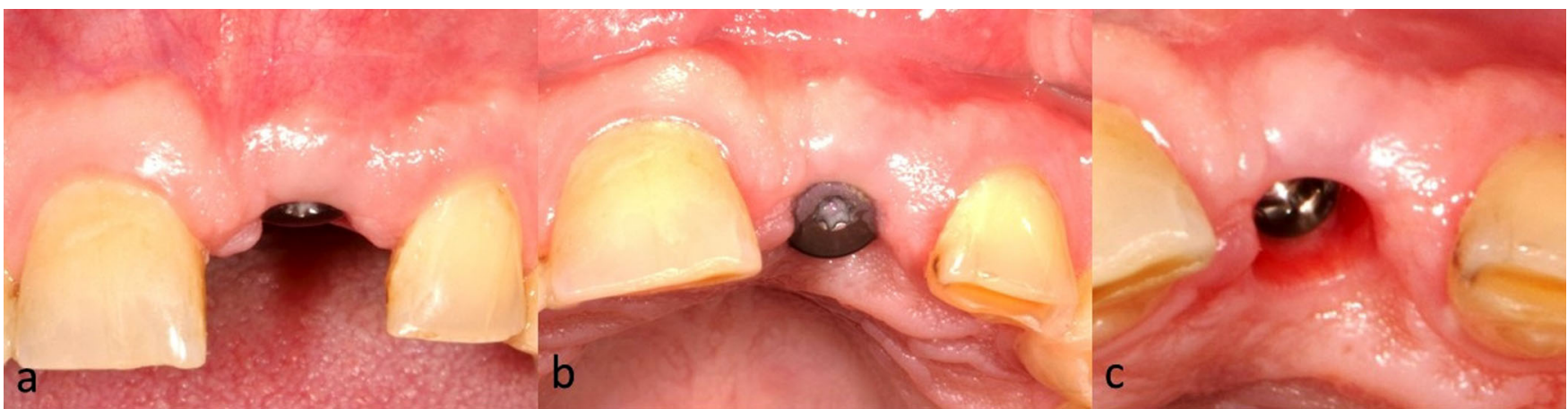

The final implant born restoration was individually fabricated on a Ti-base (Variobase®) according to the digital project. (Fig. 10). The emergency profile of the restoration was created accordingly to the shape of the adjacent central incisor.

Fig. 10. Digital planning and fabrication of the final crown.

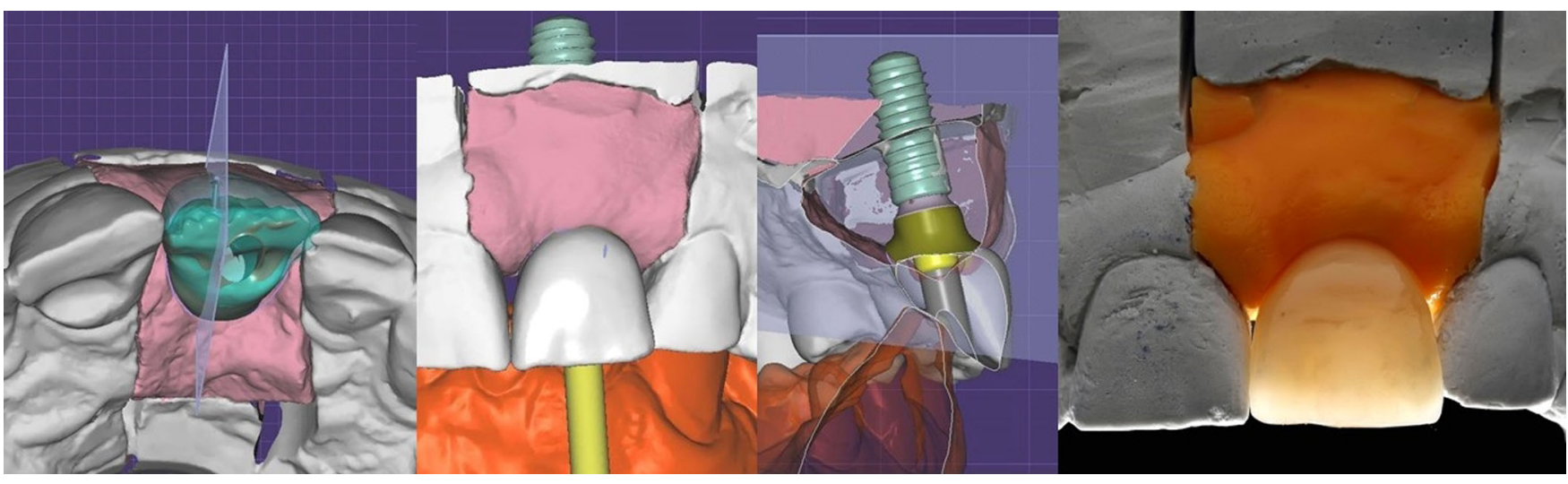

\section{RESULT:}

The application of this newly developed by the author modification of the surgical protocol allowed precise implant placement consistent with the biological width requirements and simultaneously achieving buccal gingival augmentation with complete preservation of the soft tissues in the area, thus creating good prerequisite for a proper aesthetic result. The final prosthetic restoration fulfilled the aesthetic and functional criteria required for successful long term result (Fig. 11).

Fig. 11. Placement of the final restoration.
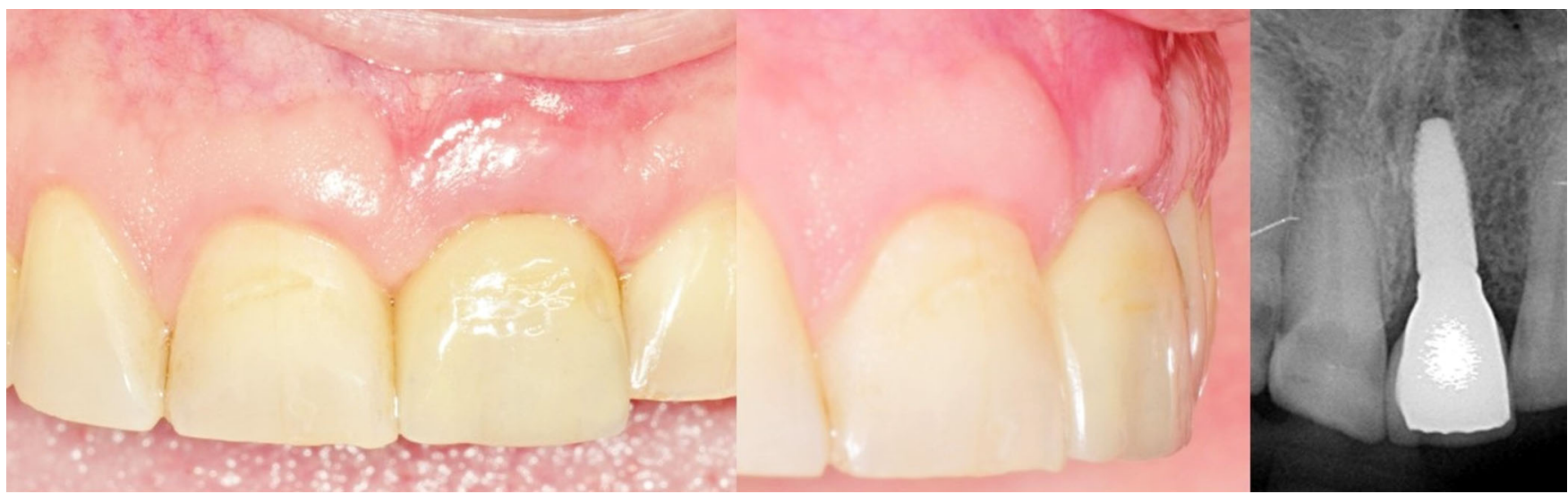

\section{DISCUSSION:}

Postextractional ridge collapse is a common clinical challenge in contemporary implant dentistry. The amount of horizontal and vertical ridge loss may reach up to $60 \%$ within 2 years of tooth extraction, most of which occurs within the first year of tooth loss.

Even in the presence of an immediate implant, buccolingual width collapse of the healing extraction socket has been recorded to reach up to $4.2 \mathrm{~mm}$. This ridge loss is encountered more frequently in the absence of adequate buc- 
cal bone thickness $[1,2,6]$.

The described in the literature soft tissue augmentation techniques may satisfactorily and predictably recreate aesthetic enhancement in mild to moderate horizontal defects,

One way is tooth extraction, followed by a ridge preservation procedure, and letting the site heal for a few months. When there is a vertical deficiency, further bone grafting. After approximately 6 months the implant can be placed. If needed, soft tissue augmentation of the site can be done at implant placement or any time during the healing or provisionalization stage.

Another method of treatment is to extract the tooth and immediate implant placement with concomitant bone grafting. The deficient soft tissue can be reconstructed with connective tissue at a later time.

Many other permutations of techniques can achieve similar results. However, these approaches often require multiple surgeries to achieve an aesthetic result [3].

The contemporary conception in dentistry relies on minimally invasive approaches, avoiding surgical techniques with vast flap preparation with the ultimate goal of limiting the number of surgeries needed to achieve optimal functional and aesthetic result. Regarding mild and moderate alveolar ridge defects an often applied approaches are variations of the "Roll technique" with deepitelization and buccal folding of the crestal portion of the alveolar ridge gingiva $[4,5,6,7,8]$.
A common drawback of the diverse modification of the Roll flap is the achievement of horizontal augmentation, limiting its application to horizontal alveolar ridge defects only.

The described in this report newly developed modification of the surgical protocol combines the minimal invasive roll flap approach with the flap design for mucogingival surgery [9], thus allowing coronal flap advancement and vertical augmentation simultaneously with the horizontal provided from the roll flap.

\section{CONCLUSION:}

The proposed new surgical approach provides an opportunity for minimally invasive implant placement without periosteal elevation thus containing the integrity of the alveolar ridge and limiting the postsurgical bone resorption. The modified roll flap preserves the keratinized tissues of the alveolar ridge using them to provide adequate buccal soft tissue volume. The concomitant use of the roll flap approach with the single flap design for minimally invasive muco-gingival surgery allows coronal flap advancement and a possibility for vertical ridge augmentation. Furthermore, this approach ensures precise selection of the optimal implant depth consistent with the thickness of the soft tissue on the alveolar ridge and the intended emergency profile of the future restoration, thus providing maximal functional and aesthetic result.

\section{REFERENCES:}

1. Covani U, Bortolaia C, Barone A, Sbordone L. Bucco-lingual crestal bone changes after immediate and delayed implant placement. $J$ Periodontol. 2004 Dec;75(12):160512. [PubMed] [CrossRef $]$

2. Spray JR, Black CG, Morris HF, Ochi S. The influence of bone thickness on facial marginal bone response: Stage 1 placement through stage 2 uncovering. Ann Periodontol. 2000 Dec; 5(1):119-28. [PubMed] [CrossRef]

3. Matthews DP. The pediculated connective tissue graft: a novel approach for the "blown-out" site in the esthetic zone. Compend Contin Educ
Dent. 2008 Jul-Aug;29(6):350-2, 354, 356-7. [PubMed]

4. Abrams L. Augmentation of the deformed residual edentulous ridge for fixed prosthesis. Compend Contin Educ Gen Dent. 1980 MayJun;1(3):205-13. [PubMed]

5. Barone R, Clauser C, Prato GP. Localized soft tissue ridge augmentation at phase 2 implant surgery: A case report. Int J Periodontics Restorative Dent. 1999 Apr;19(2):141-5. [PubMed]

6. Park SH, Wang HL. Pouch Roll Technique for Implant Soft Tissue Augmentation: A Variation of the
Modified Roll Technique. Int J Periodontics Restorative Dent. 2012 Jun;32(3):e116-21. [PubMed]

7. Mali A, Kashish, Agrawal P. Soft Tissue Ridge Augmentation Using "Roll Technique"- A Case Report. Int J Scient Res. 2016; 5(9):21-23

8. Grover D, Kaur G. Soft tissue ridge augmentation using "roll technique" - A case report. IAIM. 2014 Dec;1(4): 80-85.

9. Zucchelli G. Mucogingival Esthetic Surgery. Quintessence Pub Co; 1 Slp Har/ edition. March 18, 2013. p.820.

Please cite this article as: Kotsilkov K. Modified single roll flap approach for simultaneous implant placement and gingival augmentation. J of IMAB. 2017 Jul-Sep;23(3):1667-1672. DOI: https://doi.org/10.5272/jimab.2017233.1667

\section{Address for correspondence:}

Kamen Kotsilkov, DDS, PhD -Associate Professor, Department of Periodontology, Faculty of Dental Medicine, Medical University - Sofia,

1, St. Georgi Sofiiski str., Sofia, Bulgaria.

Private Practice: Dental Clinic Kotsilkovi, 24 Vidini kuli str., Sofia, Bulgaria.

E-mail: kamenkotsilkov@gmail.com 